\title{
Probing the Molecular Environment Using Spin-Resolved Photoelectron Spectroscopy
}

\author{
G. Turri, ${ }^{1,2}$ G. Snell, ${ }^{1,2}$ B. Langer, ${ }^{3}$ M. Martins, ${ }^{4}$ E. Kukk, ${ }^{5}$ S. E. Canton, ${ }^{1,2}$ R. C. Bilodeau, ${ }^{1}$ N. Cherepkov, ${ }^{6}$ J. D. Bozek, ${ }^{2}$ \\ A. L. Kilcoyne, ${ }^{2}$ and N. Berrah ${ }^{1}$ \\ ${ }^{1}$ Department of Physics, Western Michigan University, Kalamazoo, Michigan 49008, USA \\ ${ }^{2}$ Advanced Light Source Division, Lawrence Berkeley National Laboratory, Berkeley, California 94720, USA \\ ${ }^{3}$ Max-Born-Institute, Max-Born-Strasse 2A, 12489 Berlin, Germany \\ ${ }^{4}$ Institut für Experimentalphysik, Universität Hamburg, Luruper Chaussee 149, D-22761 Hamburg, Germany \\ ${ }^{5}$ Department of Physical Sciences, P.O. Box 3000, University of Oulu, FIN-90014 Oulu, Finland \\ ${ }^{6}$ State University of Aerospace Instrumentation, 190000 St. Petersburg, Russia
}

(Received 3 March 2003; published 8 January 2004)

Angle- and spin-resolved photoelectron spectroscopy with linearly and circularly polarized synchrotron radiation were used to study the electronic structure of model triatomic molecules, hydrogen sulfide, and carbonyl sulfide. The spin-polarization measurements of the molecular field split components of the S $2 p$ photolines revealed a strong effect of the different molecular environments. The validity of simple atomic models to explain the results is discussed.

PACS numbers: 33.15.-e, 33.60.-q, 33.80.-b

Energy and angle-resolved photoelectron spectroscopy have been widely used to study the electronic structure of atoms, molecules, and condensed matter as well as the dynamics of the photoemission process. However, the information that can be obtained with this technique is often insufficient to completely and uniquely describe the photoionization process, even when the simplest systems are investigated [1]. Various techniques have been introduced to enhance the information available from these experiments. Some detect coincidences between an electron and other electrons [2], ions [3], or fluorescence photons [4,5], while other methods utilize the alignment of the atom or molecule under investigation [6]. Spinresolved electron spectroscopy provides an alternative means to acquire an expanded data set for the photoionization of atoms and molecules. Spin-resolved innershell photoionization and Auger decay have been investigated in detail for atoms [7-10]. For molecules, only the outer shell spin-resolved photoionization of HI [11] and $\mathrm{HBr}$ [12] have been reported previously. Spinresolved electron spectroscopy has also been used to study the electronic structures of adsorbates [13], thin films [14], and the magnetic properties of solids [15].

Inner-shell photoemission from molecules is considerably more complex than the atomic case owing to the lower symmetry of the system which leads to the additional splitting of core levels and to a large number of outgoing partial waves. In addition, there is also the vibrational degree of freedom which is absent in atoms. The experimental energy resolution required to resolve these molecular effects is considerably more stringent than that required for atomic measurements. Theoretical treatments similarly require simplifying approximations to make the calculations tenable, sometimes to the detriment of the results.

In this work, we have used for the first time spinresolved $\mathrm{S} 2 p$ photoelectron spectroscopy to measure the effects of the molecular environment in carbonyl sulfide (OCS) and hydrogen sulfide $\left(\mathrm{H}_{2} \mathrm{~S}\right)$. Both molecules are of great interest in biology and astrophysics as well as from a fundamental point of view $[16,17]$. The two molecules have different molecular geometries around the sulfur atoms: OCS is linear with a terminal sulfur atom and $\mathrm{H}_{2} \mathrm{~S}$ is bent with a central sulfur atom. Highresolution spectroscopy has previously been used to study S $2 p$ excitation and ionization in both OCS [18-20] and $\mathrm{H}_{2} \mathrm{~S}$ [18]. In both cases, the nonspherical field induced by the molecular geometry around the sulfur atom removes the degeneracy of the $\mathrm{S} 2 p_{3 / 2}$ level, resulting in a splitting in the photoelectron spectrum. The magnitude of this molecular field splitting is well reproduced by a simple molecular model $[18,19]$. Calculated angle-integrated photoionization probabilities are also in good agreement with the measurements [18,19].

Kukk et al. [20] recently studied the angular distribution of S $2 p$ electrons photoemitted from OCS to probe the effect of the molecular environment. The two molecular field split components of the S $2 p_{3 / 2}$ photoline were found to have different angular distributions, and a semiempirical atomic model was employed to explain the results. The present study is motivated in part by these previous results, since a comparison between the spinresolved and spin-integrated angular distributions can yield additional insight into the effects of the molecular field on inner-shell photoionization.

The spin-resolved measurements were performed at the Advanced Light Source (ALS) using an elliptically polarizing undulator (EPU) at beam line 4.0.2 [21]. A photon flux of approximately $10^{12}$ photons/s at a resolving power $E / \Delta E$ of about 1000 was used. The EPU was set to deliver $100 \%$ circularly polarized light. Electron energy analysis was performed using a time-of-flight (TOF) spectrometer, collecting electrons emitted at $45^{\circ}$ with respect to the plane of the storage ring, in the plane 
perpendicular to the photon propagation direction. A spherical Mott polarimeter of the Rice type, operated at $25 \mathrm{KV}$, mounted at the end of the TOF was used to carry out the electron spin-polarization analysis $[22,23]$. The geometry of the experiment was selected to measure the polarization of the spin component of the electrons along the photon propagation direction. Instrumental asymmetries of the Mott polarimeter were eliminated by reversing the helicity of the photon beam approximately every $10 \mathrm{~min}$ and later recombining the results. Spin-resolved $\mathrm{S}$ $2 p$ photoelectron spectra were obtained at six photon energies between 180 and $260 \mathrm{eV}$, with the $\mathrm{S} 2 p$ threshold at about $170 \mathrm{eV}$ for both molecules [18]. Within this energy range, the photoelectrons are emitted with kinetic energy lower than the sulfur $L M M$ [24] and $L V V$ [25,26] Auger electrons. The spin-resolved measurements were complemented by high-resolution measurements of the angular distribution of the $\mathrm{S} 2 p$ photoelectron in $\mathrm{H}_{2} \mathrm{~S}$. The same experimental apparatus and conditions used by Kukk et al. [20] were chosen, with the only difference that $\mathrm{Kr} 3 d$ lines were used for the calibration of the analyzer efficiency.

Results of the spin-resolved measurements in OCS obtained with $205 \mathrm{eV}$ photon circularly polarized light are given in Fig. 1. The spin unresolved photoelectron spectrum is shown in the bottom frame of the figure, corresponding to the sum of spectra measured with left and right circularly polarized light. Data have been converted from flight time to kinetic energy and grouped at intervals of $50 \mathrm{meV}$. The shape of the $\mathrm{S} 2 p$ photoelectron spectrum is determined by three factors: the spin-orbit splitting of the S $2 p$ hole, vibrational excitations in the residual ion, and molecular field splitting of the $S 2 p_{3 / 2}$ hole. To resolve the different components of the peaks, we applied a least-squares fitting procedure to the experimental spectra (solid and dashed lines in Fig. 1) utilizing

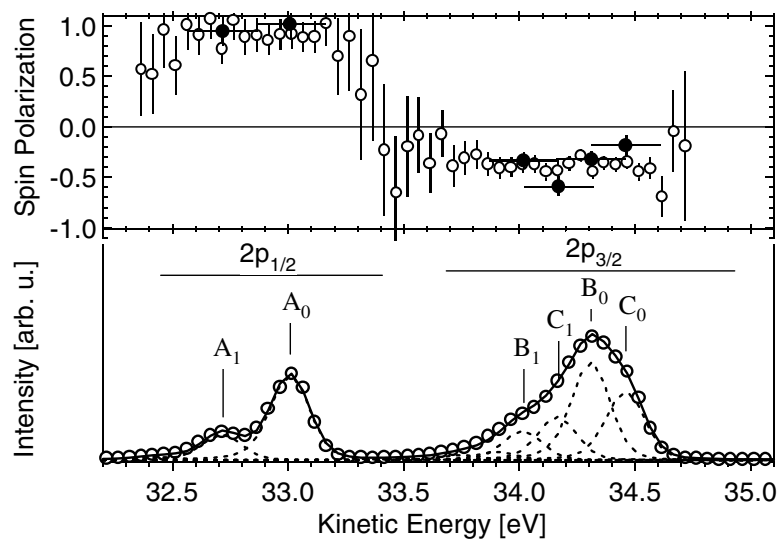

FIG. 1. S $2 p$ photoionization spectrum of OCS, measured with $205 \mathrm{eV}$ circularly polarized light. Bottom: total intensity; the continuous curves are the result of a least-squares fitting procedure. Top: spin polarization; calculated from $(\mathbf{O})$ the peak areas and $(\bigcirc)$ the individual data points. asymmetric Voigt functions. Asymmetric distortion of the line shape due to postcollisional interaction was included using the method of van der Straten et al. [27]. Previously determined values of the lifetime width (65 meV), vibrational period (279 $\mathrm{meV})$, and molecular field splitting (145 meV) [20] were used in the fit. The areas of the peaks obtained from the fitting procedure were used to determine the spin polarization for the individual photoelectron lines as described below. Additionally, we performed a single channel analysis [10], where the spin polarization was determined for each point of the spectrum. The results of the two methods are compared in the top panel of Fig. 1 (solid and empty circles, respectively) and are in good agreement. The error bars of the spin-polarization values in the figure include both statistical and systematic errors.

The photoelectron spectrum of $\mathrm{H}_{2} \mathrm{~S}$ is similarly determined by spin-orbit, vibrational, and molecular field splittings. Values of the vibrational splitting (about $300 \mathrm{meV})$, molecular field splitting $(110 \mathrm{meV})$, and $\mathrm{S} 2 p$ lifetime width $(70 \mathrm{meV})$ from Refs. $[18,19]$ were used in the fits of the spectra to extract peak areas. In the following discussion of the results, we concentrate on transitions leading to the ground vibrational state of the ion, labeled as $A_{0}, B_{0}$, and $C_{0}$ in Fig. 1.

The spin polarization of the three $\mathrm{S} 2 p$ lines are depicted as a function of photon energy in Figs. 2 and 3 for OCS and $\mathrm{H}_{2} \mathrm{~S}$, respectively. In our notation a positive spin polarization indicates that the electrons are preferentially emitted with spin antiparallel to the photon propagation direction. The angular distribution of photoelectrons with definite spin orientation can be expressed by five parameters, namely, the total cross section $\sigma$, the anisotropy parameter $\beta$, and three parameters related to the spin polarization, $A, \gamma$, and $\eta$ (we are adopting the

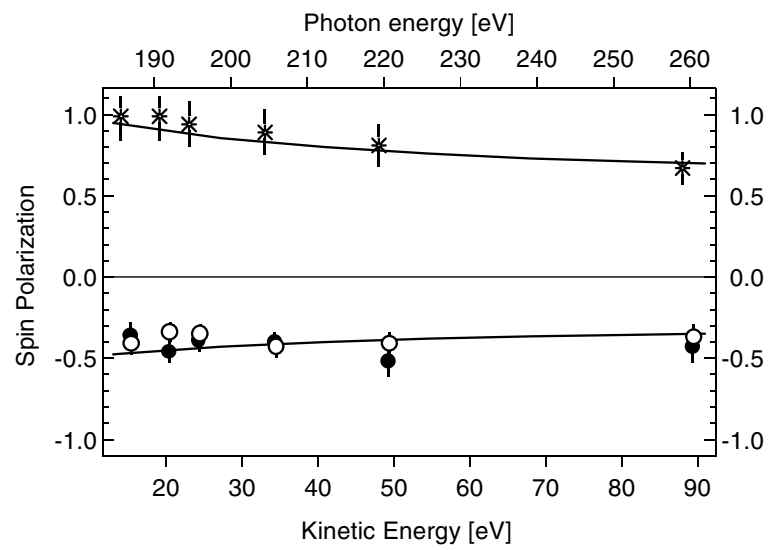

FIG. 2. Spin polarization of the $\mathrm{S} 2 p$ photoelectrons in OCS as a function of (circularly polarized) photon energy. Experimental points are for the ground vibrational state of the $\left(*=A_{0}\right) \mathrm{S} 2 p_{1 / 2}$ and $\left(-=B_{0}, \bigcirc=C_{0}\right)$ molecular field split $\mathrm{S} 2 p_{3 / 2}$. Solid lines are calculations for $\operatorname{Ar} 2 p$ photoionization. 


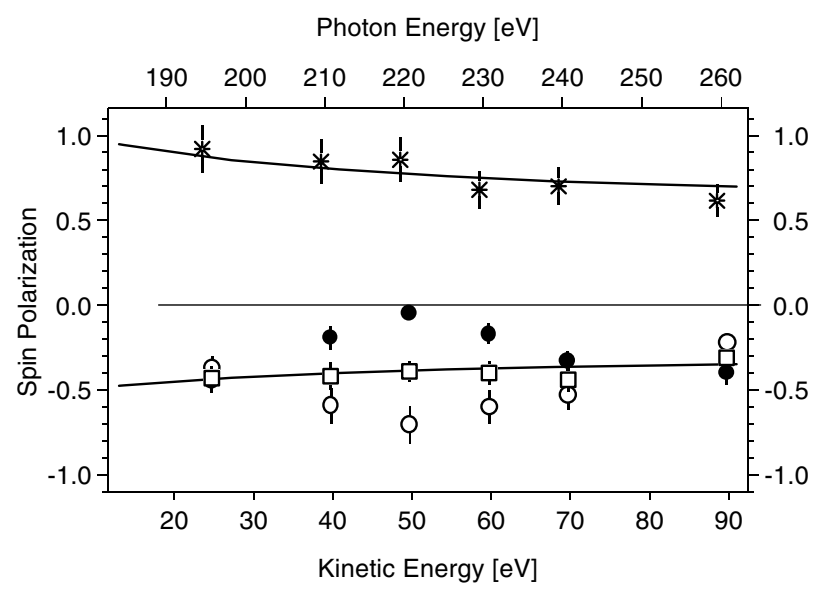

FIG. 3. Spin polarization of the $\mathrm{S} 2 p$ photoelectrons in the $\mathrm{H}_{2} \mathrm{~S}$ molecule as in Fig. 2. The spin polarization of the $(\square)$ sum of the $\mathrm{S} 2 p_{3 / 2}$ lines is also reported.

notation used by Cherepkov $[28,29])$. In the geometry of our experiment, the spin polarization takes the simple form [28]

$$
P=\frac{-(A+\gamma / 2) S}{1+\beta / 4}
$$

where $S= \pm 1$ is the photon helicity.

Calculations of the parameters in Eq. (1) are not trivial for molecules. In reality, the initial molecular state is a linear combination of orbitals with different orbital angular momenta, and an infinite number of partial waves are allowed for the electrons in the continuum. In order to explore the effects of the molecular environment, we start by describing the process within a fully atomic model, for which calculations can be easily performed. Using an atomic model is a valid approximation because of the localized nature of the S $2 p$ core orbitals. Only one initial state is then allowed, the electron can be emitted only as an $s$ or a $d$ wave (neglecting the electron spin-orbit interaction in the continuum), and the process is described by two matrix elements, S and D, respectively, and by a phase shift, $\delta_{s d}$. In both molecules, the sulfur is in the electronic configuration of Ar. Because the photoionization cross section for the $2 p$ levels of second row elements depend only weakly on the nuclear charge, calculations for Ar can be used. Calculations of the spin polarization for Ar $2 p$ photoionization have been published previously by Cherepkov [28] and are reproduced in Figs. 2 and 3 as solid lines. In the case of $\mathrm{S} 2 p_{1 / 2}$ photoionization, giving rise to line $A_{0}$, which is a single nondegenerate level and therefore cannot be split by the molecular field, the spin polarization is correctly reproduced for both molecules by the atomic calculation. The situation is very different for the S $2 p_{3 / 2}$ lines $B_{0}$ and $C_{0}$. The atomic model seems to correctly reproduce the spin polarization of the lines $B_{0}$ and $C_{0}$ in OCS, but not those of $\mathrm{H}_{2} \mathrm{~S}$ which deviate from one another over a wide energy range. However, if the unresolved $\left(B_{0}+C_{0}\right)$ lines are considered (empty squares in Fig. 3), then again the $\mathrm{Ar}$ calculations are able to reproduce the spin polarization of the lines in $\mathrm{H}_{2} \mathrm{~S}$.

A similar behavior can be observed in the measured angular distributions. We report in Fig. 4 the anisotropy coefficient $\beta\left(B_{0}+C_{0}\right)$ of the unresolved $B_{0}+C_{0}$ lines and the difference in the anisotropy coefficients of the two lines $\beta\left(C_{0}\right)-\beta\left(B_{0}\right)$ as we measured in $\mathrm{H}_{2} \mathrm{~S}$ (empty circles and empty triangles, respectively). These are compared with the values of $\beta\left(B_{0}+C_{0}\right)$ and $\beta\left(C_{0}\right)-\beta\left(B_{0}\right)$ measured by Kukk et al. [20] in OCS (solid circles and solid triangles, respectively) and with the results of $\mathrm{Ar}$ calculations (solid lines). For both molecules, the Ar calculations correctly reproduce $\beta\left(B_{0}+C_{0}\right)$ in the $195-$ $300 \mathrm{eV}$ photon energy range, whereas the agreement is less satisfactory around the minimum of $\beta\left(B_{0}+C_{0}\right)$. In contrast, the difference in the anisotropy coefficients of lines $B_{0}$ and $C_{0}$, which cannot be accounted by a pure atomic model, is observable over a broad energy range. Thus, from the measured values of the spin polarization and the anisotropy coefficients it appears that the effects of the molecular environment tend to vanish when the experiment integrates over the different orientations of the $2 p$ angular momentum, restoring somehow the spherical symmetry. This behavior is consistent with the strong atomic character of the $\mathrm{S} 2 p$ orbital in these molecules as well as the absence (or low oscillator strength) of shape resonances above their $L_{23}$ sulfur edges $[16,30]$.

Kukk et al. [20] extended the atomic model to account for the molecular effects by assuming that the same matrix elements, but different phases, are associated with lines $B_{0}$ and $C_{0}$. The anisotropy parameter $\beta$ is proportional to the cosine function of the phase shift difference $\delta_{s d}[20,28]$, and a fast variation of $\delta_{s d}$ in one of the channels associated to lines $B_{0}$ and $C_{0}$ can produce

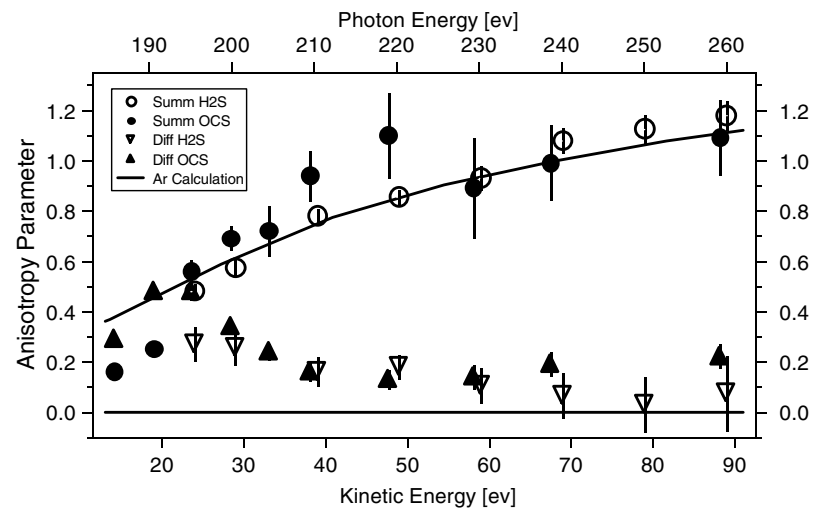

FIG. 4. Anisotropy parameters of the unresolved doublet $B_{0}+C_{0}\left[(\bigcirc) \mathrm{H}_{2} \mathrm{~S}\right.$, (O) OCS [20]] and difference in the anisotropy coefficients of the two lines $\left[(\nabla) \mathrm{H}_{2} \mathrm{~S},(\boldsymbol{\Delta})\right.$ OCS [20]]. Ar calculations are reported as solid lines. 
a strong effect. But the degree of polarization (1) after the substitution of parameters $A$ and $\gamma$ [28] is proportional to the same cosine function, so that it is expected to have a similar effect in both the anisotropy parameter $\beta$ and the degree of polarization $P$. Then the vanishing difference of the spin polarization between the two lines, $B_{0}$ and $C_{0}$, in OCS as reported here would require a vanishing difference in the anisotropy parameters $\beta$ under the above model, in contrast with the experimental results of Kukk et al. [20]. Also in $\mathrm{H}_{2} \mathrm{~S}$ the difference between the spin polarization of the $B_{0}$ and $C_{0}$ lines shown in Fig. 3 is not accompanied by a difference of the corresponding $\beta$ parameters shown in Fig. 4. Thus, in order to describe both the angular distribution and the spin polarization of the two lines, different matrix elements must be employed for lines $B_{0}$ and $C_{0}$. A full understanding of the different behavior of lines $B_{0}$ and $C_{0}$ in the two molecules would require detailed calculations, which are beyond the scope of this Letter. We can suggest only that different behavior for the photoelectron lines in the two molecules is connected with the different structure of these molecules, due to which the ligand field split levels are characterized by different quantum numbers. As a result, transitions to states $B_{0}$ and $C_{0}$ in these molecules obey different selection rules, which leads to a difference in the behavior of all parameters.

In summary, spin-resolved electron spectroscopy was combined with angular distribution measurements to measure the spin polarization and the anisotropy parameter of S $2 p$ electrons from OCS and $\mathrm{H}_{2} \mathrm{~S}$ for the first time. Differences in the electron spin polarization for the molecular field split $\mathrm{S} 2 p_{3 / 2}$ lines of $\mathrm{H}_{2} \mathrm{~S}$ but not in OCS highlight the sensitivity of this differential technique to the molecular field. This work also tested the applicability of simple atomic models and identified a way to delineate molecular intricacies that could be used in general for other systems.

This work has been funded by DOE, Office of Science, Office of Basic Energy Sciences, Chemical Sciences, Geosciences and Biosciences Divisions. The ALS is sup- ported by the Director, Office of Science, of the U.S. Department of Energy under Contract No. DE-AC0376SF00098. We thank E. Arenholz, A. Young, and B. Rude for their support at the ALS beam lines 4.0.1 and 10.0.1. E. Kukk acknowledges the financial support from the Research Council for the Natural Sciences of the Academy of Finland.

[1] B. Schmidtke et al., J. Phys. B 33, 5225 (2000).

[2] K. Ueda et al., Phys. Rev. Lett. 83, 5463 (1999).

[3] E. Shigemasa et al., Phys. Rev. Lett. 74, 359 (1995).

[4] H.-J. Beyer et al., J. Phys. B 28, L47 (1995).

[5] K.W. Mclaughlin et al., Phys. Rev. Lett. 88, 123003 (2002).

[6] O. Plotzke et al., Phys. Rev. Lett. 77, 2642 (1996).

[7] U. Heinzmann, J. Phys. B 13, 4353 (1980).

[8] U. Heinzmann, Appl. Opt. 19, 4087 (1980).

[9] G. Snell et al., Phys. Rev. A 66, 022701 (2002).

[10] G. Snell et al., Phys. Rev. Lett. 82, 2480 (1999).

[11] N. Böwering et al., J. Phys. B 24, 4793 (1991).

[12] M. Salzmann et al., J. Phys. B 27, 1981 (1994).

[13] G. Schönhense et al., Phys. Rev. Lett. 56, 512 (1986).

[14] P. G. Steeneken et al., Phys. Rev. Lett. 88, 047201 (2002).

[15] M. Maiti et al., Phys. Rev. Lett. 88, 167205 (2002).

[16] R. Feng et al., Chem. Phys. 252, 359 (2000).

[17] V.G. Tyuterev et al., Chem. Phys. Lett. 348, 223 (2001).

[18] M. R. F. Siggel et al., J. Chem. Phys. 105, 9035 (1996).

[19] S. Svensson et al., Phys. Rev. Lett. 72, 3021 (1994).

[20] E. Kukk et al., J. Phys. B 33, L51 (2000).

[21] A.T. Young et al., Nucl. Instrum. Methods Phys. Res., Sect. A 467, 549 (2001).

[22] G. C. Burnett et al., Rev. Sci. Instrum. 65, 1893 (1994).

[23] G. Snell et al., Rev. Sci. Instrum. 71, 2608 (2000).

[24] T. X. Carroll et al., J. Electron Spectrosc. Relat. Phenom. 51, 471 (1990).

[25] F. Gel'mukhanov et al., Phys. Rev. A 53, 1379 (1996).

[26] C. M. Truesdale et al., J. Chem. Phys. 80, 2319 (1984).

[27] P. van der Straten et al., Z. Phys. D 8, 35 (1988).

[28] N. A. Cherepkov, J. Phys. B 12, 1279 (1979).

[29] N. A. Cherepkov, J. Phys. B 14, 2165 (1981).

[30] H. Nakamatsu et al., J. Chem. Phys. 95, 3167 (1991). 